\title{
Design of Wastewater Treatment Control System Based on Siemens PLC
}

\author{
Lubin Zhao, Tong Zhao \\ College of Automation and Electronic Engineering, Qingdao University of Science and Technology, \\ Qingdao, China \\ 492768939@qq.com
}

Keywords: sewage treatment, Siemens PLC, WinCC configuration software

\begin{abstract}
With the rapid development of the national economy, PLC-based automatic control technology has been widely used in industrial production. The automatic control system consists of Siemens PLC S7-300 module, CPU315-2PN/DP as the main control unit, the indicators of sewage of all workshops are monitored and all blowers, lift pumps, and switch valves are monitored and controlled. The upper computer software WinCC can be used to monitor and control in real time the parameters of the height, $\mathrm{pH}$, temperature, oxygen content and other parameters of all the sewage treatment tanks. At the same time, all the sewage data can be archived and displayed in the form of historical curves. The company's monitoring and treatment of production wastewater in this way can greatly improve the efficiency and quality of wastewater treatment, and reduce the impact of human factors on the quality of wastewater treatment in the wastewater treatment process.
\end{abstract}

\section{Introduction}

The second branch factory sewage treatment and control system has completed the effective monitoring and control of the entire sewage treatment process. According to the change of the control physical quantity in each stage of the sewage treatment, the corresponding processing actions can be automatically completed according to the set plan. In this way, the indicators of sewage treatment can be maintained to meet the requirements set by users. At the same time, it is closely integrated with the wastewater treatment process to meet the requirements of the wastewater treatment plant for management and safe treatment, automatic control and alarm of the production process, automatic protection, automatic operation, automatic adjustment, improvement of operating efficiency, reduction of operating costs, and reduction of labor Intensity, on-line real-time monitoring of the computer's on-line and real-time monitoring of key parameters and equipment operating conditions of the various processes in the wastewater treatment plant to ensure that the effluent quality of the wastewater treatment plant meets the design emission standards.

\section{Control System Design}

Sewage treatment automation control system is based on PCS7 software development, running on WINXP operating system platform, HMI human-machine interface, adopting the way of 
dual-machine running independently and back-to-back, and display the process parameters on the man-machine interface in the form of data overview, flow chart, curve, alarm window, etc. According to the principle of process relevance, the screen design function of WinCC is used to organize related parameter designs into the same picture. Through the means of one-button switching, all parameter browsing can be easily switched. The upper computer monitor adopts WinCC software and mainly completes the monitoring of real-time data such as DO, PH, liquid level, and flow, fault alarm, time and date, status display and control of electrical equipment, login rights management, and trend analysis. Combined with the control of the lower computer PLC, a set of complete automation control system was designed. The CPU315-2PN/DP served as the main control unit, using ET200M distributed remote I/O to realize the decentralized management and centralized control of all sewage treatment pools. The combination of software and hardware makes the entire system more reliable, and enables functions such as sequential start and stop, fault chain stop, and heavy fault emergency stop.

\subsection{The hardware design}

The PLC operates as a system control unit to monitor and control the entire process of the system control. The superiority of the PLC host controller is closely related to the reliability, safety, production efficiency, and production cost of the overall system operation. Therefore, it is very important to select a suitable PLC host controller. Siemens CPU315-2PN/DP integrated DP master-slave interface and MPI interface, has strong communication capabilities, easy to implement the distributed structure, user-friendly interface, flexible operation mode, with great room for upgrade, It can accomplish more complex tasks without adding any hardware. PROFIBUS and PROFINET communication make communication network easy, modular programming makes programming more flexible and can solve a variety of complex tasks.

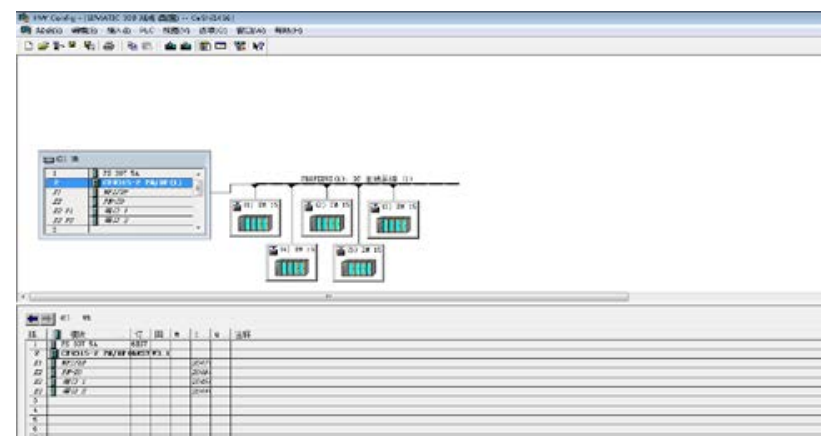

Fig.1 Hardware topology diagram

This design uses distributed I/O to directly control distributed equipment around the site, and uses PROFIBUS-DP communication between host CPUs for information transmission. Among all distributed I/Os, the ET200M can configure all the modules of an S7-300 PLC, making it easy to use, efficient, and more scalable for more complex tasks. The Siemens PLC replaced the traditional relay electrical control hardware, greatly reducing the installation and wiring of the control cabinet. Through the PLC developer can carry on the simulation and the debugging of the user program in the laboratory, can shorten the electrical engineer's design and the debugging time greatly. In terms of equipment maintenance, due to the very low failure rate of Siemens series PLCs, the maintenance workload of the equipment is also very small; And Siemens PLC has its own hardware self-diagnosis function, in the debugging process can be based on the instructions on the PLC and its own diagnostic fault information on troubleshooting the debugging process, greatly facilitates the maintenance. The PLC control system mainly includes three parts: a central processor, signal 
input units (such as: switches, buttons, and detection sensors), and output execution units (such as contactors, solenoid valves, and signal indicators). The input unit of the control system is connected to the input point of the PLC, and the digital instruction signal and the status signal of the controlled object are input to the PLC through the input point. The output execution unit is connected to the PLC output to drive the controlled object to work. The hardware system topology is shown in Figure 1.

\subsection{The software design}

The sewage treatment control system in this design is different from the traditional sewage treatment system. The control is through the preparation of the PLC program to fully control the equipment in the system and complete the precise sewage treatment tasks. On the basis of satisfying the basic monitoring, the remote control of field devices in the automatic mode, including the functions of the timed-on device, cycle-on device, and cycle-on device, greatly reduces the on-site operator's labor intensity, improves the efficiency, and reduces the equipment loss. The upper computer monitoring software prepared by WinCC has the functions.

(1) Control, monitoring, parameter setting, fault alarm, and fault diagnosis functions for the equipment and operation results in the entire system.

(2) According to the requirements of the sewage treatment process and logic, strictly control the start, stop, fault or emergency stop of the equipment.

(3) Dynamic display of the system. The screen displays the various process flows intuitively on the screen, allowing real-time monitoring of the operating status and parameters of the system equipment.

(4) Choose a reasonable automatic operation mode so that the operation of the entire system is in a safe and stable state, and it can meet the technical requirements for sewage treatment.

(5) When the equipment or control parameters are close to an abnormal state, the interface has an alarm and is accompanied by an audible alarm.

(6) The alarm history record is made, the fault classification is filed, and the alarm record cannot be deleted for accident analysis and recall.

(7) Historical data is stored in the history database. Operators can easily generate running reports and curves for important equipment and monitoring indicators, and have the ability to call and print at any time.

(8) The system has a user rights management function, and has different levels of authority for each key operation of the project to ensure the security of the system.

(9) The system has a remote monitoring (network monitoring) function. The management personnel can view the operation status of the sewage treatment site in real time through Ethernet. The specific software structure is shown in Figure 2.

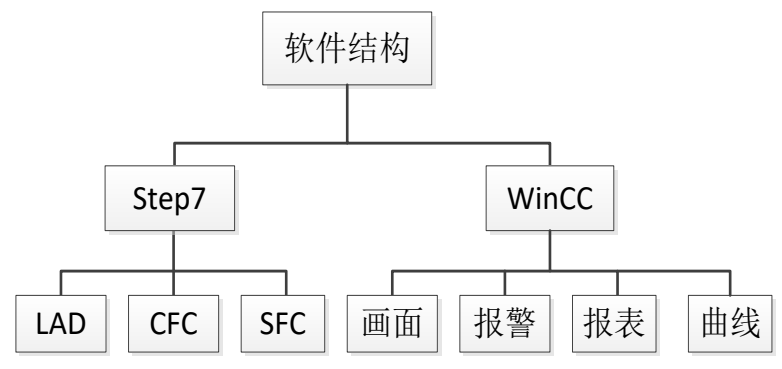

Fig.2 Software structure 


\subsection{Communication program design between PLC}

In the entire automation system, the communication program part between the master station and the substation occupies a very important position. The communication between the master station and the substation uses the PROFIBUS address of the substation ET200M module and the substation address defined in the hardware configuration as the communication module identifier. The physical connection uses a purple profibus-DP line to connect five substations in series. A 330 $\Omega$ terminating resistor is added to the last substation to eliminate signal reflections in the communication cable during communication. In order to carry on the data transmission, here need to call FC5 "AG-SEND" and FC6 "AG-RECV" module, to send and receive data. At the same time, the FC105 value conversion module must be called to convert the collected field data and store it in the corresponding DB block before it can be displayed in WinCC. The key to the physical layer of Ethernet communication lies in that the IP address must be set in the same network segment, and at the same time pay attention to the influence of the subnet mask on the network segment. Here, the network segment is unified on the 192.168.0.X interval to ensure that the communication on the physical layer is unblocked. The process of establishing the connection is not complicated, and the correct configuration and IP allocation can be used. The transmission of data can be based on the M zone, DB block, etc. Flexible use of different transmission methods can achieve a variety of different control methods. The communication network structure is shown in Figure 3.

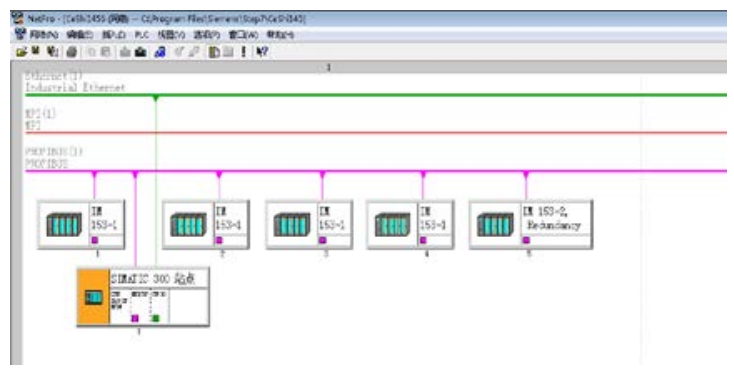

Fig.3 Network program structure

\subsection{Host computer WinCC screen design}

The upper computer uses the Siemens configuration software WinCC to monitor the design of the screen, mainly to complete $\mathrm{DO}, \mathrm{PH}$, liquid level, flow and other real-time data monitoring, fault alarm, date and time, electrical equipment status display and control, login rights management, trend analysis. The operator can intuitively understand the scene through the screen. The specific design steps are detailed.

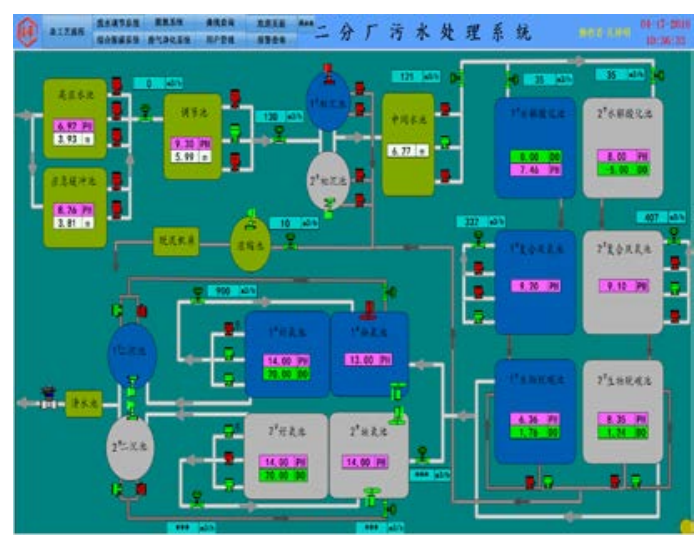

Fig.4 WinCC monitor screen 
(1) Create a WinCC monitor screen as shown in Figure 4. The host computer control system has a total of seven main monitoring screens: total process flow chart, wastewater control system screen, integrated decarburization system screen, denitrification system screen, exhaust gas purification system screen, alarm query screen, and history curve screen.

(2) Create internal variables. Open the "internal variable" under the variable management of the WinCC project manager, right-click and select "new variable group", then open the created variable group, right-click on "new variable" and name the new variable. Finally set the data type.

(3) Create process variables. Process tags are used for communication between WinCC and the automation system. On the basis of satisfying the physical connection, the WinCC server and the automation system (such as a PLC) need to establish a communication driver connection that matches the PLC model, and set the attributes of the channel connected with the PLC.

(4) Connection variables. The graphic object in the graphic editor needs to join the smart object input and output fields for establishing the connection relationship with the variables. After the variables are connected, in the system display screen, the graphic objects such as pneumatic valves and lift pumps will change their colors according to the data changes of the variables. The feed pipe will also show the effect of flow, and the input and output fields of $\mathrm{PH}$ value, liquid level, and flow rate will also update the corresponding data as the reaction progresses. The operator can monitor the status of the sewage treatment process accurately by monitoring the data transmitted to the computer from the scene and displaying the changes in the color of the graphics on the monitoring screen, which achieves the purpose of remote monitoring of the computer.

\section{Implementation of Sewage Treatment Monitoring Function}

In the design and research of the entire sewage treatment system, the status parameter display, switch operation, and emergency stop pump should be regarded as the key design and implementation part. In the process of manual sewage treatment, the overflow of the liquid level, the inaccuracy of the physical parameters, and the low efficiency of the sewage treatment often occur. In order to better improve these issues, radar level gauges, acid-alkaline monitors, and flow meters are used to help achieve accurate processing when designing automatic material control systems. With the advantages of convenient, quick and sensitive WinCC screens, the personnel of the Ministry of Environmental Protection can effectively, quickly and accurately control all the sewage valves and lift pumps in real time, and the PLC outputs signals to the valves and pumps to turn them on and off. The liquid level, $\mathrm{pH}$ value, and sewage pipeline flow of each treatment tank are displayed on the monitoring screen of WinCC using a radar level gauge, an acid alkaline monitor, and a flow meter.

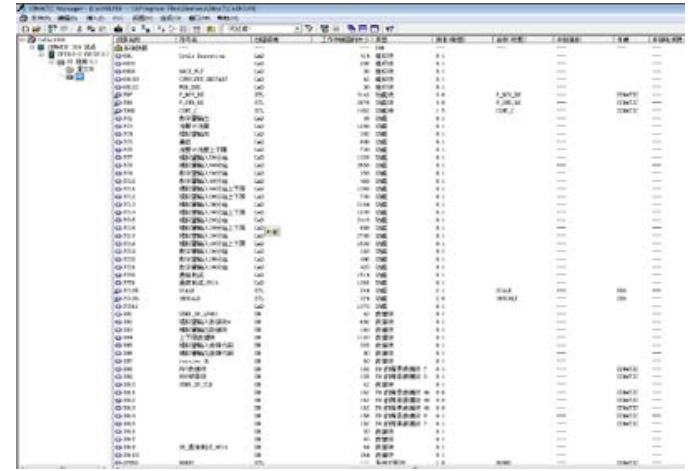

Fig.5 LAD ladder program

(1) The entire sewage treatment control system should be based on the LAD ladder program. In 
this project, the ladder diagram mainly implements three functions: First, it converts all the analog data collected in the field, and displays it on the WinCC screen; the second is that the communication program is to realize the MODBUS polling communication of the on-site partial flowmeter. The third is to start and stop the control of all lift pumps and blowers on the site. Variable frequency motors are frequency-controlled at different frequencies. The ladder program is shown in Figure 5.

(2) Monitor the status of each sewage treatment tank on the computer in the operation room, including the operation status of the sewage lifting pump, the switch status of each pipeline valve, and the physical parameters such as liquid level, oxygen content, and $\mathrm{PH}$ value in the sewage treatment tank. If the line valve is open, the background color of the valve on the screen will turn green, and the background color of the off status will display red. The background color of the lift pump is the same as the line valve, green when open, and red when closed. If the lift pump and blower malfunction, the background color of the corresponding pump or blower in the display will turn yellow.

(3) In the wastewater adjustment screen, the $\mathrm{PH}$ value and temperature value of the waste water to be treated in each workshop can be monitored in real time. If the temperature and $\mathrm{PH}$ value are outside a reasonable range, the staff can intuitively see and take measures for the corresponding treatment. After the workshop waste water enters the regulating tank, it needs the staff to detect and control its liquid level and PH value, and the treated sewage can enter the next level of hydrolysis acidification tank for other treatments. The real-time monitoring screen is shown in Figure 6.

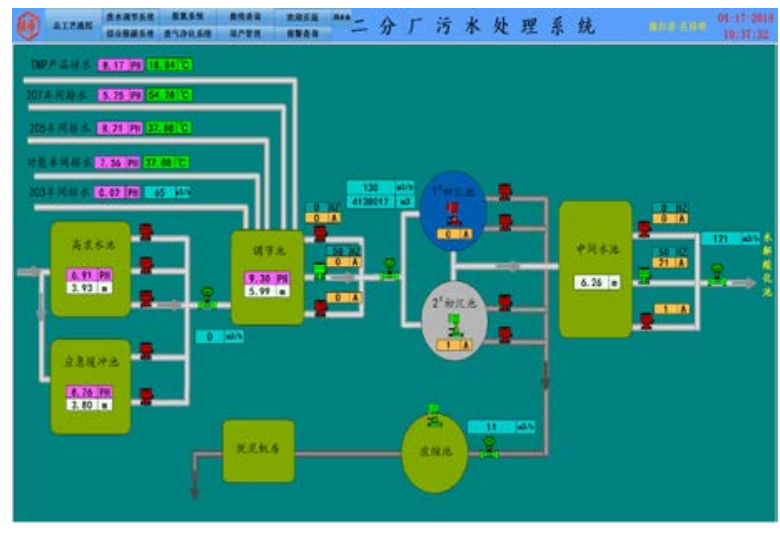

Fig.6 Real-time monitoring screen

(4) All screens can be switched by one button, and the screen switching function can be realized by setting the parameters in the "event" and "mouse action" options in the buttons of each screen. The screen switch is shown in Figure 7.

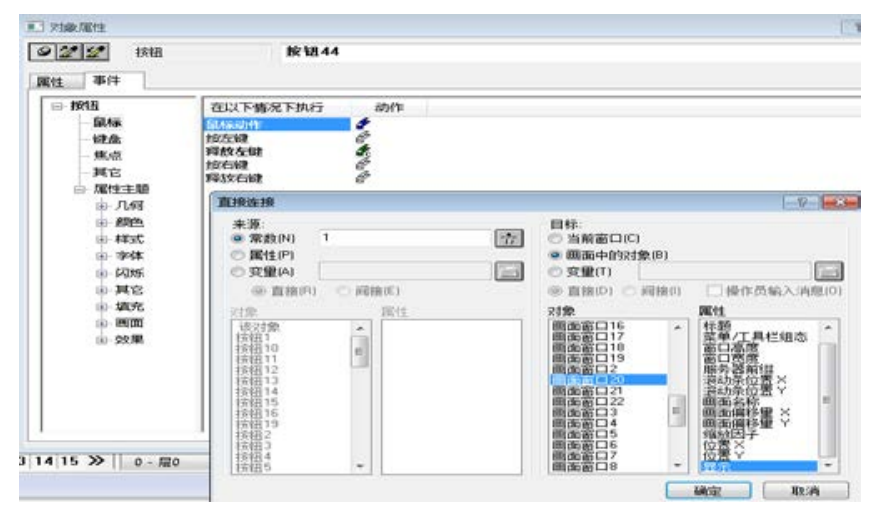

Fig.7 Screen switching 
(5) The user management authority setting is mainly to ensure the normal operation of the entire system, to avoid random people and other free access to the operation screen to avoid danger. The operator enters the operation screen and needs to input the login user name and password. Only the correct password can be entered. User rights can be changed and set by the engineer, and other people do not have permission to modify it. The login interface is shown in Figure 8.

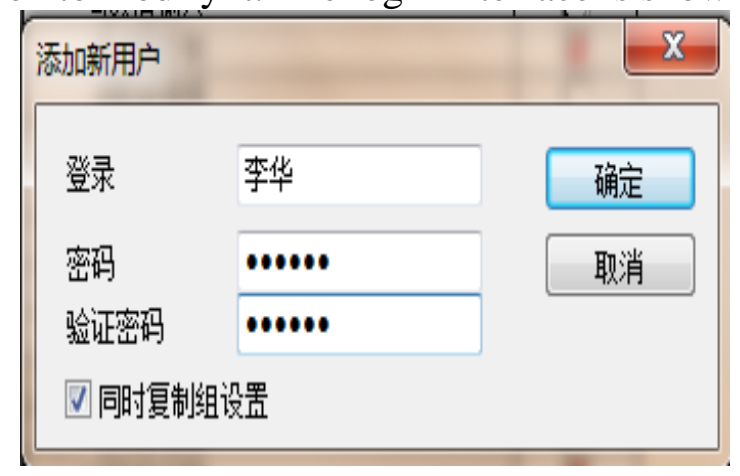

Fig.8 Login interface

(6) The alarm inquiry screen displays and archives the alarm condition of the entire system by adding an alarm control. The operator can call up the alarm record at any time for viewing. The alarm control in WinCC can have a detailed record of the alarm conditions in each phase of the past. The alarm message text shows the alarm content. The background is red indicating that there is an alarm event, the background is green indicating that the alarm event disappears, and the background is blue indicating that the alarm event has been confirmed. Alarm records are shown in Figure 9.

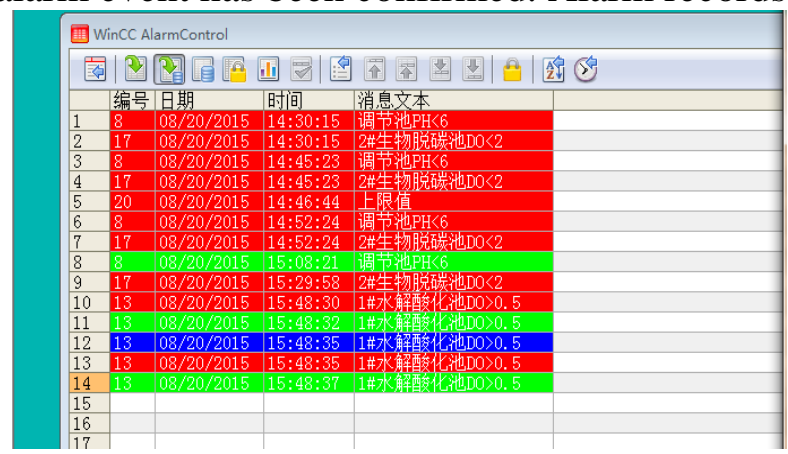

Fig.9 Alarm record

\section{Conclusion}

The control system designed in this paper is based on Siemens S7-300 PLC and configuration software WinCC sewage treatment control system. The upper computer software WinCC can be used to monitor and control in real time the parameters of the height, $\mathrm{pH}$, temperature, and oxygen content of all sewage treatment tanks. At the same time, all sewage data can be archived and displayed in the form of historical curves. The company's monitoring and treatment of production wastewater in this way can greatly improve the efficiency and quality of wastewater treatment, and reduce the impact of human factors on the quality of wastewater treatment in the wastewater treatment process. Through the production test, the control accuracy of this control system can fully meet the design requirements of the system, greatly enhance the degree of automation in chemical production, greatly improve the accuracy of wastewater treatment, improve the efficiency of the use of human labor and equipment production efficiency, and achieve good results in production. 


\section{References}

[1] Yao Baiying. Design of domestic sewage control system based on plc control. Suzhou University, 2010.

[2] Cang Bingnan, Li Xiaosheng, Wang Qingkai. Water treatment plant full flow automatic control system based on S7-300 and WinCC.(Mineral processing section), 2016,vol 2,pp.85-89.

[3] Ji Jiangong. Design of Distributed Control System for Wastewater Treatment Based on WinCC [D].Beijing University of Chemical Technology, 2008.

[4] Cao Dongchao. Siemens PLC's Automation Control System Design for Municipal Wastewater Treatment Plants.Ecological and Environmental Engineering, 2013, vol.3, pp.203-204.

[5] Wang Xin. Research and Design of pH Value Based on plc for Wastewater Treatment Control System and Water Quality.Taiyuan University of Technology, 2015.

[6] Su Dongsheng. Design of control system based on plc wastewater treatment system.Mechanical and electrical engineering technology, 2013, vol.42 (4), pp.65-68.

[7] Zhang Bo. Design of Wastewater Treatment Control System Based on plc and Kingview. He Bei University, 2012. 